\title{
A Novel Approach for Shock Absorber----Colloidal Damper
}

\author{
Muchun $\mathrm{Yu}^{1, \mathrm{a},{ }^{*} \text {, Zhiling Niu}}{ }^{1, \mathrm{~b}}$, Peng Zhao ${ }^{2, \mathrm{c}}$ and Zijun Zhang ${ }^{1, \mathrm{~d}}$ \\ ${ }^{1}$ China Academy of Launch Vehicle Technology, Beijing 100076, China \\ 2State Key Laboratory of Mechanics and Control of Mechanical Structures, Nanjing University of \\ Aeronautics and Astronautics, Nanjing 210016, China \\ amcyu89@126.com, bnethulip@163.com, cpzhao_detec@nuaa.edu.cn, dche_guevara@163.com
}

Keywords: Colloidal damper, shock absorber, hydrophobic nanoporous materials, energy dissipation.

\begin{abstract}
Using the mixture of nanoporous materials and its associated lyophobic fluid for damping application is a novel technology called colloidal damper. Compression caused liquid intrusion transfers the mechanical energy into interface energy and so that producing damping effect and colloidal damper has great potential as shock absorber. In this paper, a colloidal damper consist water and hydrophobic silica gel particles as working medium is investigated. The working mechanism of colloidal is first introduced. Then, an experiment is conducted to investigate the mechanical properties of colloidal damper. Finally, the energy dissipation performance is analyzed.
\end{abstract}

\section{Introduction}

Hydraulic damper is a shock absorber or damper device of which viscous liquid flows through narrow interstices to dissipate energy. Although widely used, there exist some disadvantages of hydraulic damper, such as complicated structures and friction hearting which may change the viscous of working fluid.

Complementary to Hydraulic damper, colloidal damper is proposed by Eroshenko[1]. Eroshenko try the mixture of solid porous material and its associated lyophobic fluid in mechanical engineering for mechanical application. And now it has been one of the most novel research fields. This novel technology takes advantage of the theory of water molecules intrusion and extrusion nanopores to store, release and dissipate mechanical energy. Considering whether a hysteresis occurs during the loading-unloading procedure, the solid-liquid mixture can be used either as colloidal damper [2,3] or undamped molecular spring $[4,5]$.

The colloidal damper consists of a piston-cylinder structure (fig. 1) and nanoporous functional material which is usually the mixture of hydrophobic silica gel particles and water. The piston of colloidal has no damping holes. The silica gel particles are silica with massive connected or unconnected nanopores modified by hydrophobic chemical groups. The modified pores gain hydrophobicity so that they resist water from intrusion till the liquid pressure is higher enough to overcome the capillary pressure (fig. 2). Though both utilizing the principle of liquid flow through narrow orifices, colloidal damper is very different with conventional hydraulic damper. For a colloidal damper, water intrudes into nanopores when the nanoporous functional material is compressed instead of holes in millimeter range. Hence, mechanical energy is transfer into surface energy rather than dissipated by heart for a colloidal damper [6].

Compared with hydraulic damper, colloidal damper exhibits the following advantages: it is able to absorb more mechanical energy per unit quantity of working material, without heart or with very limited heart; the restoring force is not depend on the velocity; it has wide working frequency $(0 \sim 30 \mathrm{~Hz})$ and it has higher efficiency [2]. Due to these important advantages, colloidal damper can be employed for spacecraft recovery, aircraft landing-gear, vehicle suspensions, and various anti-vibration protections, etc.

It is deserve to be mentioned that hydrophobic silica gel particles and water are not the only mixture for colloidal damper. Actually, any solid porous matrix and its associated lyophobic fluid are 
potential to produce a colloidal suspension. The solid porous matrix could be silica gel, aerogel, ceramics, glass, zeolites, carbon (graphite, charcoal, fullerenes, and carbon nanotubes), aluminum oxide, etc [1,7]. The associated lyophobic fluids are water, mercury, melted metals, melted flux or melted salts, etc. Nevertheless, silica gel particles and water are the most popular mixture because it is easily accessible and environmental-friendly.

In this paper, the working mechanism of colloidal damper is investigated by analyzing the process of water intrudes into and extrudes from nanopores. Then, a colloidal damper use silica gel particles and water as working substance is designed and tested to research the mechanical properties of colloidal damper. Finally, the energy dissipating performance of colloidal damper is also discussed.

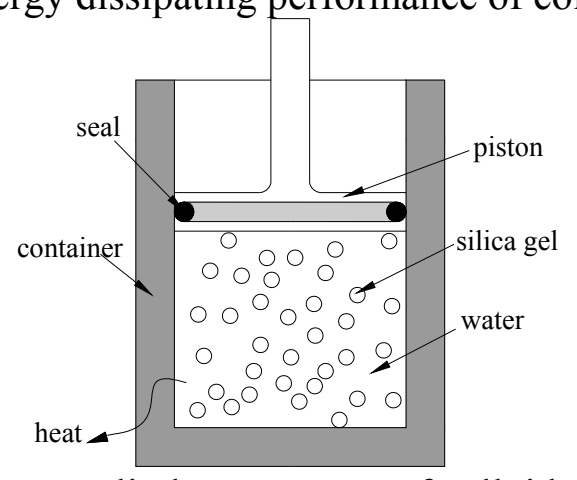

Fig. 1 Piston-cylinder structures of colloidal damper

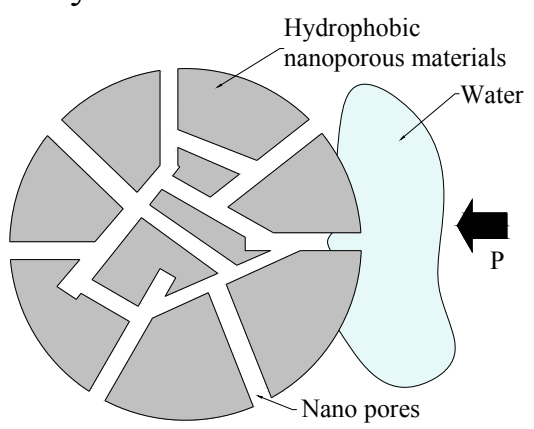

Fig.2 Water intrudes into hydrophobic nanopores

\section{Working mechanism}

The key mechanism of colloidal damper is water intrusion and extrusion from nanopores[1]. The difference of intrusion and extrusion pressure brings a hysteresis to the loading-unloading curves.

The Laplace capillary pressure describes the additional force generated by hydrophobic pore walls as the following equation

$$
P_{c}=-\frac{2 \sigma \cos \theta}{r}
$$

of which $P_{c}$ is the capillary pressure, $\sigma$ is surface tension of water, $\theta$ is the contact angle and $r$ is the diameter of the pore. For a hydrophobic pore $\theta$ is bigger than $90^{\circ}$, consequently, the Laplace capillary pressure tend to repel water.

At compression, external hydraulic pressure works against the Laplace capillary pressure and the pressure of the gas trapped in the nanopores as observed in fig. 3. In general, for nano-sized pores, the pressure of trapped gas is negligible campared with Laplace capillary pressure[2].

For a colloidal damper, when hydraulic pressure in the cylinder is smaller than capillary pressure, water is resisted from nanopores, hence it just compresses the liquid in this stage and the stiffness is very high in this stage (Pressing water stage I). When the hydraulic pressure increases and reaches the capillary pressure, water intrudes into the nano-pores of the hydrophobic materials in a large scale, making colloidal damper very soft stiffness (Intrusion stage). After all pores have been saturated, the hydraulic pressure compresses the liquid again (Pressing water stage II). Hence, a ladder-like loading curve can be observed in the compression of colloidal damper [7]. 
At unloading, colloidal damper exhibits the reversed three stages. The liquid volume starts expansion when the pressure decrease, followed by an extrusion stage. And finally another liquid volume expansion stage occurs. However, the extrusion pressure is much lower than intrusion pressure, sometimes even lower than atmosphere pressure (in this condition, extrusion will not fully accomplished). The low extrusion pressure produces a very remarkable hysteresis so that the colloidal damper is able to dissipate mechanical energy [2].

The central issue of working principle of colloidal damper is intrusion and extrusion stages. Water intrudes into hydrophobic pores in the intrusion stage, leading to a large volume change. According to equ.(1), if the size and the contact angles of the pores are constant, water intrudes into the pores all at once at the critical pressure and all pores get saturated. Then the force-displacement curve of the intrusion stage will exhibit a horizontal plateau. However, in practice, the pores of nanoporous materials vary both in size and hydrophobicity, depending on material properties. So water molecules first fill the pores that have the largest apertures and the smallest contact angles and then fill the smaller pores with larger contact angles and so on till all pores are filled with water under external pressure.

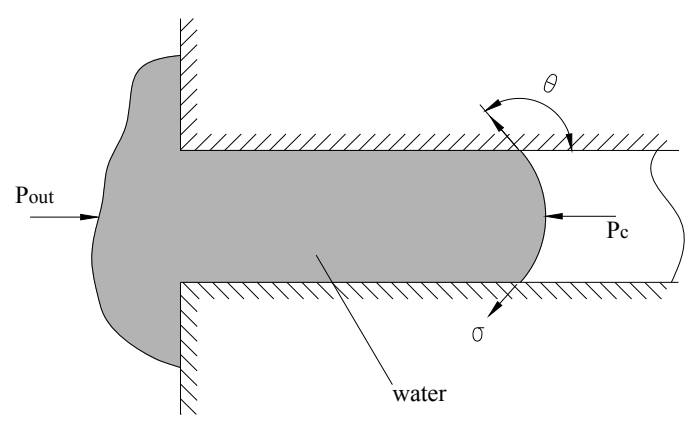

Fig. 3 Water intrudes into a hydrophobic pore

The extrusion follows the same principle i.e. Laplace capillary equation, with the intrusion stage. The difference between intrusion pressure and extrusion pressure is attributed to contact angle hysteresis, which occurs for a rough surface or a chemical heterogeneous surface [6]. When the contact angle is dynamically measured, one can observe different values of advancing angle $\theta_{\mathrm{a}}$ and receding angle $\theta_{\mathrm{r}}\left(\theta_{\mathrm{a}}>\theta_{\mathrm{r}}\right)$ as shown in fig. 4 . The hysteresis angle $\theta_{\mathrm{h}}=\theta_{\mathrm{a}}-\theta_{\mathrm{r}}$ may be several degrees or several tens of degrees, depending on the surface roughness and chemical inhomogeneities. Consequently, the Laplace capillary pressure of intrusion and extrusion stages varies.
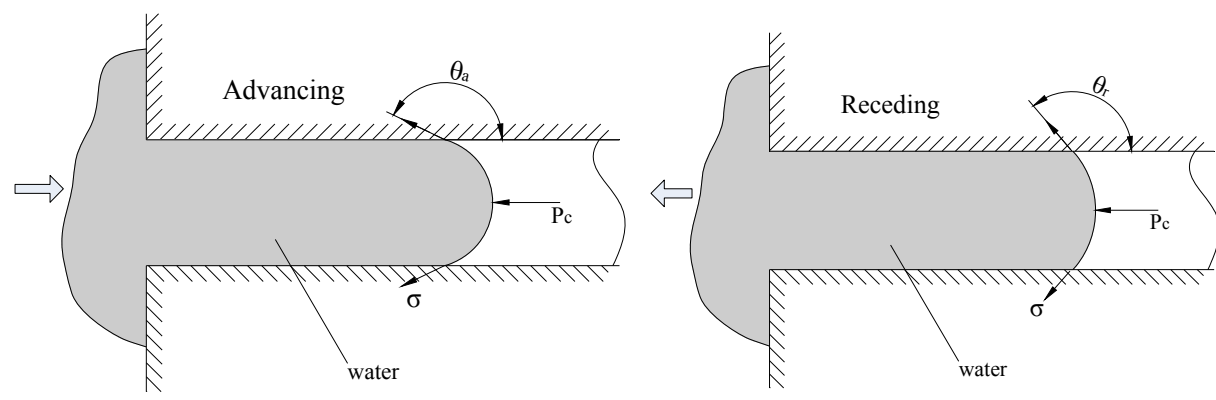

Fig. 4 contact angle hysteresis

In compression, the volume change includes the saturated pores volume and compressed volume of liquid. The compressed volume of liquid is proportional with pressure. The relationship of external pressure and saturated pores volume can be derived as follows: When the pressure rises to one value, the pores whose Laplace capillary pressure is smaller than this value are saturated. Combine with the statistical parameters of pores' diameters and contact angles, the volume change can be obtained by integrating the saturated pores' volume. Mechanical model of compression can be established 
following reference [9], which investigated the working principle of molecular spring. The relationship of volume change and pressure can be calculated using the same method.

\section{Experiments}

An experiment is conducted to investigate the loading-unloading mechanical properties of colloidal damper. The mixture of $5 \mathrm{~g}$ commercial hydrophobic silica gel particles and water are sealed in a piston-cylinder structure to compose a colloidal damper. The mean diameter of the silica gel particles is $50 \mu \mathrm{m}$ as shown in fig. 5 and the mean pore diameter is $12 \mathrm{~nm}$. The silica gel particles are modified by linear chain of 18-alkylchlorosilanes $(\mathrm{C} 18)$ to obtain hydrophobicity. The piston-cylinder structure is composed of a $10 \mathrm{~mm}$-diameter piston and a $24 \mathrm{~mL}$ compressible chamber. The loading-unloading load was applied and recorded using a universal test machine in fig. 6 . The loading was applied to the colloidal damper with a speed of $0.2 \mathrm{~mm} / \mathrm{s}$.
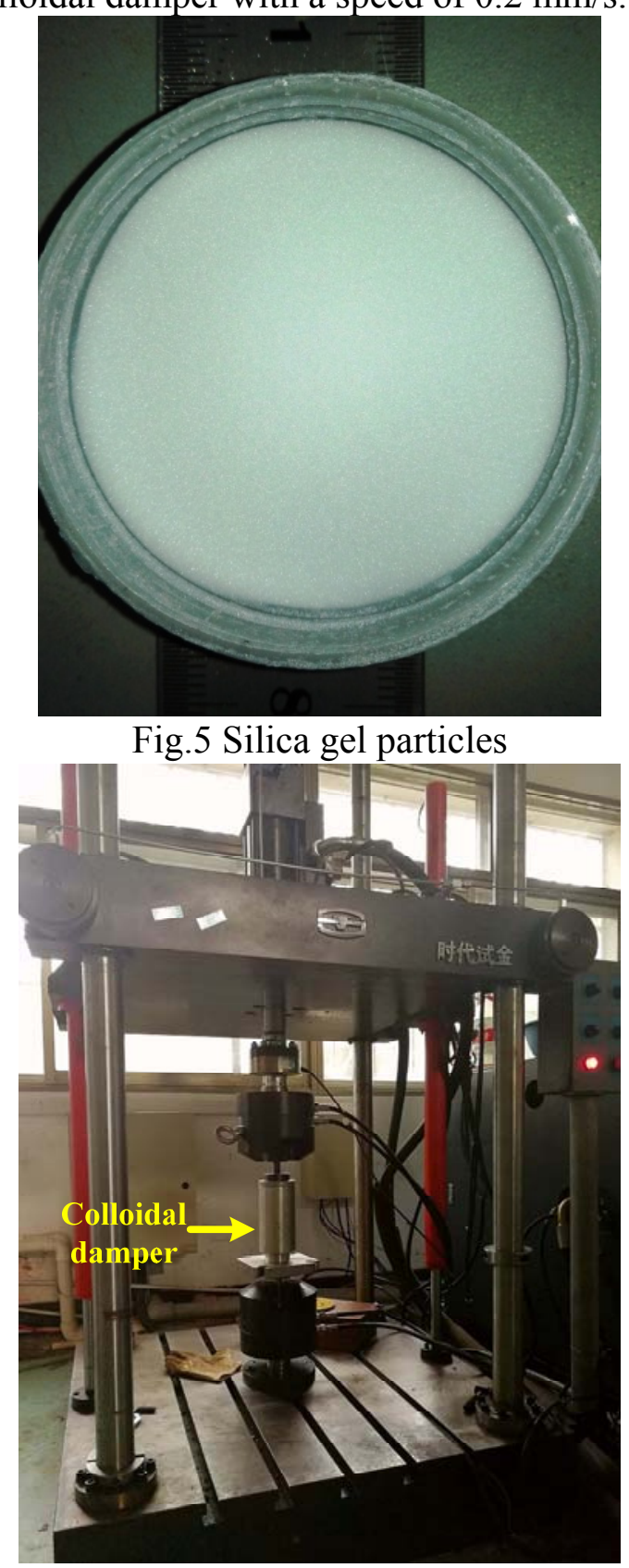

Fig.6 Colloidal damper and test rig 


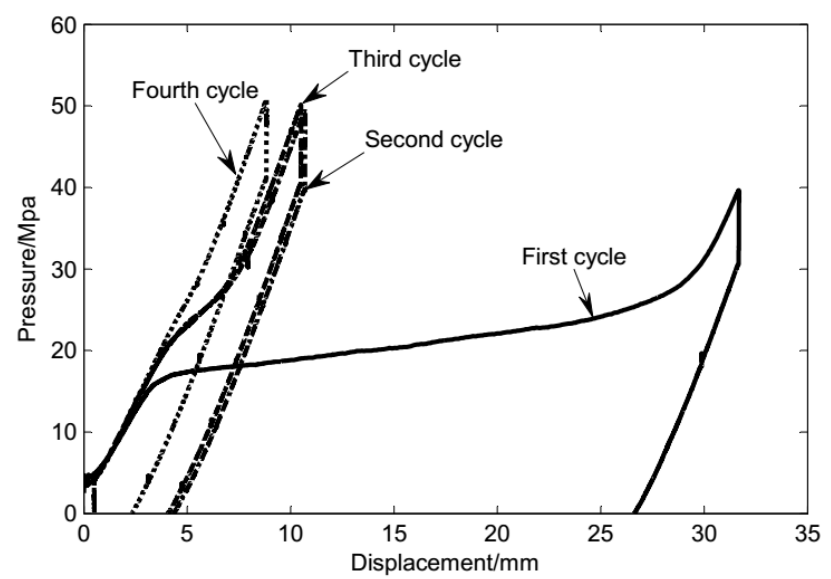

Fig. 7 Experiment measured hysteresis of colloidal damper

The measured loading-unloading curves are given in fig. 7. In this figure, irreversible cycles can be observed. A remarkable hysteresis is obtained in the first cycle, however, the hysteresises get smaller in the following cycles. For the fourth cycle, the hysteresis is even negligible. The contact angle hysteresis theory can account for the irreversible cycles. If the hysteresis angle $\theta_{\mathrm{h}}$ is large enough, although the advancing angel is larger than $90^{\circ}$, the receding angel still might be smaller than $90^{\circ}$. Hence, during the unloading procedure, for some pores whose receding angel is smaller than $90^{\circ}$, water will remain in the nanopores. Only for very hydrophobic pores (whose receding angel is bigger than $90^{\circ}$ ), the extrusion occurs. Because the non-outflow water captures the pores, the effective pore volume decrease after the first cycle. Besides, water molecules also binding with chemical defects (such as unmasked $\mathrm{OH}$ groups) on hydrophobic wall. These water molecules are trapped in the pores and make next hysteresis smaller.

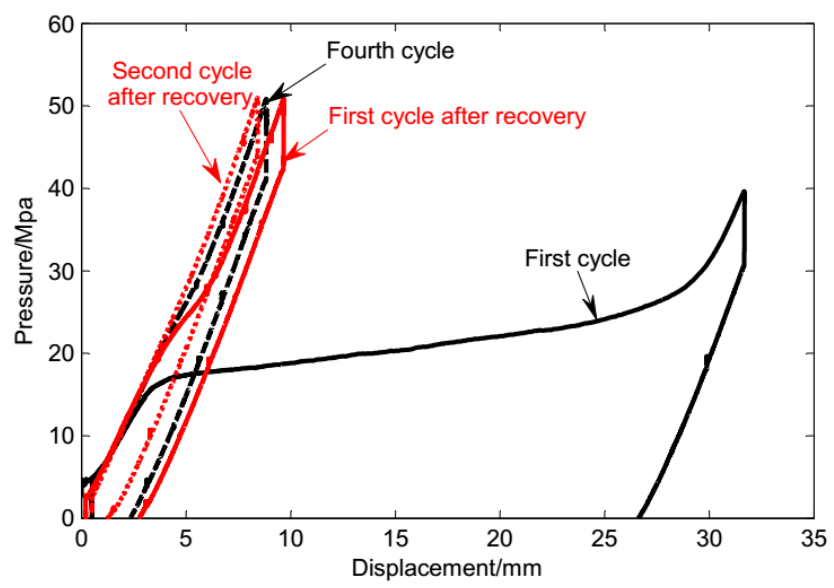

Fig. 8 Experiment measured hysteresis of colloidal damper after 20 hours recovery

After 20 hours' relaxation for recovery, we retested the colloidal damper under the same experiment conditions and the result is given in fig. 8. It can be observed that the hysteresis of the first cycle after recovery is bigger than the fourth cycle before recovery, but still smaller than the original first cycle. The second cycle after recovery is even smaller than the fourth cycle before recovery. That might because extra extrusion occurs during the relaxation. The reason is that the contact angle of the quasi-static extrusion is neither $\theta_{\mathrm{a}}$ nor $\theta_{\mathrm{r}}$, but an equilibrium contact angle as $\theta_{0}=\left(\theta_{\mathrm{a}}+\theta_{\mathrm{r}}\right) / 2$.

It also should be mentioned that at the beginning of unloading process, there is a jump of pressure. It is caused by friction force of sealing ring. More specifically, when the motion direction of piston changes, the direction of friction changes, leading to a sudden change of force.

\section{Energy dissipation efficiency}

In this section, the energy dissipation performance of colloidal damper is analyzed. An ideal damper should be able to reduce the impact force and exhibits high energy dissipation efficiency. 
Therefore, the reacting force of damper should increase sharply and keep in a platform for a certain distance. When unloading, the reacting force should decrease sharply to maintain a big hysteresis.

Fig. 9 is the first hysteresis of colloidal damper. In fig. 9, the compression curve is A-B-C-D and the decompression curve is D-E-F. The area encircled by compression curve and decompression curve corresponding to the dissipated energy $E_{\mathrm{d}}$. Meanwhile, the area under the compression curve is the mechanical work $E_{\text {a }}$ applied on the colloidal damper during a cycle. Therefore, damper efficiency (capability to dissipate the energy) $\eta$ is defined as

$$
\eta=\frac{E_{d}}{E_{a}}
$$

From the measured date of fig. 9, we can calculate that the damper efficiency of colloidal damper is $89.02 \%$ in one cycle. In comparison, the damper efficiency of a hydraulic damper is usually lower than $50 \%$ and in actual applications is about 30-40\% [2]. This result attests the excellent performance of colloidal damper.

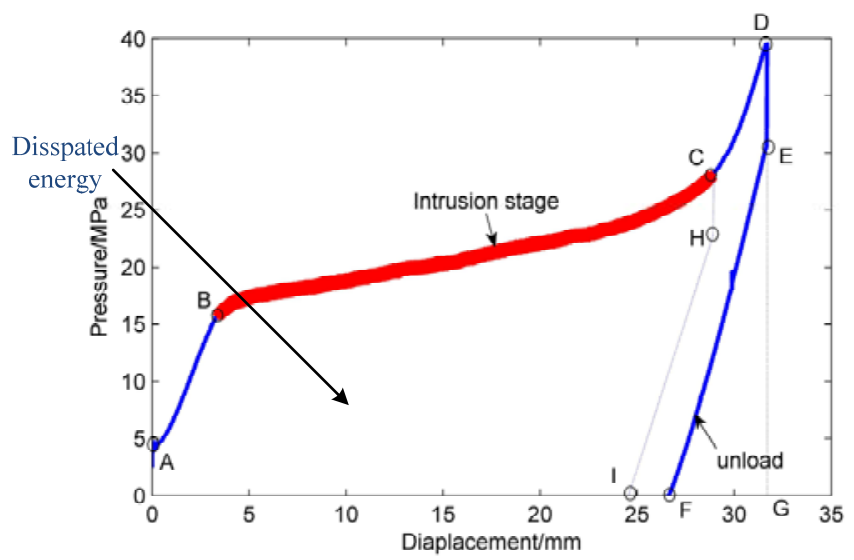

Fig. 9 Energy dissipation of colloidal damper

To reduce the impact forces mostly, the maximum displacement of colloidal damper should be confined to the intrusion stage of the lodading-unloading curve, to avoid the sharply increase of the impact force in the compression stage II. In others words, the maximum displacement should be restricted to point $\mathrm{C}$ as shown in fig. 9. Under this condition, the loading-unloading curve ought to be A-B-C-H-I, which is very close to the hysteresis of an ideal damper described before. So the impact forces can be mostly restricted. In this case, the dissipated energy is calculated to be $40.85 \mathrm{~J}$ from the data in fig. 9. Considering $5 \mathrm{~g}$ silica gel particles is filled in colloidal damper, each gram of silica gel particles is able to absorb $8.17 \mathrm{~J}$ mechanical energy. In addition, to maintain the energy absorption capacity, the colloidal damper can be designed by changing the amount nanoporous material.

Different with hydraulic damper which dissipates mechanical energy by hearting, colloidal damper transfers mechanical energy into interface energy by creating more liquid-solid interface. Hence, the colloidal damper produces very limited heart.

\section{Conclusion}

As a novel application of nanotechnology in the field of mechanical engineering, colloidal damper is investigated both on its working mechanism and its shocking absorbing performance in this paper. Though both utilizing the principle of liquid flow through narrow orifices, the energy dissipation mechanism is different between colloidal damper and hydraulic damper. For a colloidal damper, water intrusion into nanopores produces extra solid liquid interface and stores the mechanical energy as interfacial energy rather than dissipated by heart as hydraulic damper. Considering the large specific area of nanoporous materials, colloidal damper exhibits particularly high energy absorption capacity and excellent energy dissipation efficiency. The colloidal has very potential application in the field of spacecraft recovery, aircraft landing-gear, vehicle suspensions, and various anti-vibration protections, etc. 


\section{Acknowledgments}

This work was supported by the National Natural Science Foundation of China under Grant No. 11602306.

\section{References}

[1] A. Y. Fadeev, V. A. Eroshenko, Study of Penetration of Water into Hydrophobized Porous Silicas, J. Journal of Colloid \& Interface Science. 187(1997) 275-282.

[2] C. V. Suciu, T. Iwatsubo, S. Deki, Investigation of a colloidal damper, J. Journal of Colloid \& Interface Science. 259(2003) 62-80.

[3] F. B. Surani, X. Kong, D. B. Panchal, et al. Energy absorption of a nanoporous system subjected to dynamic loadings, J. Applied Physics Letters. 87(2005) 163111-163111-3.

[4] M. Soulard, J. Patarin, V. Eroshenko, et al. Molecular spring or bumper: A new application for hydrophobic zeolitic materials, J. Studies in Surface Science \& Catalysis. 154(2004) 1830-1837.

[5] A. Ryzhikov, I. Khay, H. Nouali, et al. High pressure intrusion-extrusion of electrolyte solutions in aluminosilicate FAU and *BEA-type zeolites, J. Microporous \& Mesoporous Materials. 221(2016) 1-7.

[6] C. V. Suciu, T. Iwatsubo, K. Yaguchi, et al. Novel and global approach of the complex and interconnected phenomena related to the contact line movement past a solid surface from hydrophobized silica gel, J. Journal of Colloid \& Interface Science. 283(2005) 196-214.

[7] A. Han, V. K. Punyamurtula, Y. Qiao. Effects of post-processing treatment on sorption isotherm of an effectively hydrophilic zeolite, $\beta$, J. Philosophical Magazine Letters. 88(2008) 37-42.

[8] M. C. Yu, X. Gao, Q. Chen. Study of the Mechanical Properties and Vibration Isolation Performance of a Molecular Spring Isolator, J. Shock \& Vibration. 2016, 2016:1-10.

[9] M. C. Yu, Q. Chen, X. Gao. Theoretical and experimental investigation of molecular spring isolator, J. Microsystem Technologies. 23 (2017) 285-292. 